\title{
Scheduling design flow of armored vehicle with Tabu search
}

\author{
Jianjun Lin ${ }^{a}$, Dong $\mathrm{Yu}^{\mathrm{b}}{ }^{\mathrm{b}}$, Lu Jia ${ }^{\mathrm{c}}$ \\ China North Vehicle Research Institute, Beijing 100072, China \\ a11267216@qq.com, bdysunshine@126.com, 'clujia@noveri.com.cn
}

Keywords: Tabu search, knowledge engineering platform, German Leopard.

\begin{abstract}
We deal with resource constrained project scheduling problems (RCPSP for short) in this study, whose aim is to build knowledge platform framework for enterprise. We take the transmission design of German leopard 2 tanks as an example, construct a classic RCPSP design example, and then apply the tabu search to address this issue. The experimental results verified the effectiveness of the proposed tabu search.
\end{abstract}

\section{Introduction}

Recently domestic enterprises are facing with the inheritance of the previous knowledge and research result, and the creation of knowledge engineering platform could solve this problem. Knowledge engineering platform is based on the integration of knowledge of process and design, combined with knowledge discovery, knowledge management, domain ontology and knowledge innovation, etc. Meanwhile, the existing knowledge of enterprise is teased and managed, and also is pushed and reused in the process of research. Moreover, the valuable data in the process of research could be concluded and summarized to form new knowledge and thus to realize the presentation of the right knowledge to the right people at the right time, and ensure the accomplishment of research tasks [1].In the light of the study on the knowledge platform framework and utilization of enterprise, this paper studied the transmission design issue of classic German leopard 2 tanks RCPSP problems.

At present, numerous intelligent optimization algorithms has been widely applied to the classic RCPSP problems in recent years. A series of tasks in the project usually could use the directed network with a Node type (Activity on Node, AON) to represent [2].In AON network, each node represents a task and the arrows between nodes are to represent the logical relationship between the tasks. In the classic RCPSP, network is not allowed to appear in circulation and feedback.

\section{Introduction to RSPSP issue}

RCPSP problems are closely linked with the engineering practice which is complex, a wide range of scholars have conducted the special research on this issue, the classic RCPSP mainly involves two kinds of constraints, one is about logic and another is resource constraints. Project goal for scheduling problems includes the minimum project time [3], minimum average delay [4], the minimum number of delay tasks, the maximum net present value and the minimum average weighted time flow [5], etc.

The classical RCPSP model can be described as follows [6]:

Target: $\min C T_{j}$

s.t.:

$C T_{h} \leq S T_{j}, \quad \forall h \in P_{j}$

$\sum_{j \in I_{t}} r_{j}^{k} \leq r^{k}, \forall k \in R, t \geq 0$

Where, formula 2.1is the target function, means the minimum project schedule, formula 2.2 is the task logic end, formula 2.3 is the resource constrains. 


\section{Classic RCPSP problem solving based on tabu search algorithm}

Tabu Search (TS) is a kind of Global optimization of the intelligent algorithm. The general process of tabu search can be describe as flows:

Step1: Set the parameters, generate the initial solution, blanking tabu list

Step2: using the current solution to generate the neighborhood solution

Step3: Select the candidate solution

Step4: Judge the Amnesty guidelines, if eligible, choose the best solutions as the current solution, or choose the noon-tabu solution.

Step5: Judge the termination condition, if eligible, print results, or turn to step2.

It's flow chart and pseudo code show as follows:

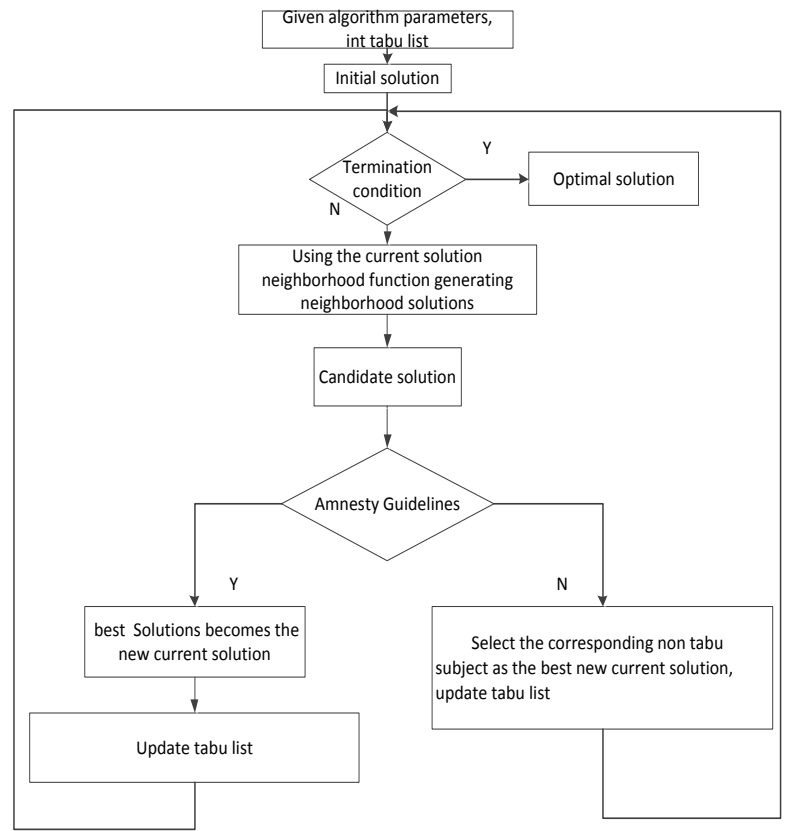

Flowchart

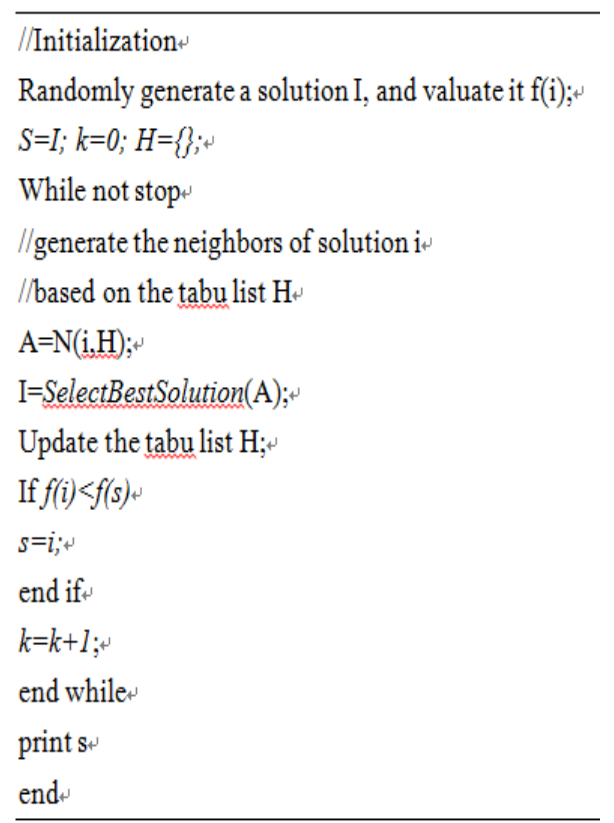

(b) Pseudo code

Fig.1 Flowchart and Pseudo code of tabu search

In order to verify the effectiveness of tabu search algorithm in classic resource-constrained scheduling problem (RCPSP), this paper take German Leopard II tanks' transmission design to construct a numerical example, and use Tabu search algorithm for solution. Take the shortest period as the optimized target, according to the previous resource constraints, the project scheduling problem can be described by classical RCPSP problem, apply the PWW model[7], we can obtain a mathematical model of this problem as:

Objective Function:

$$
\min \sum_{\mathrm{t}=1}^{\mathrm{D}} t x_{i t}
$$

Constrains:

$$
\begin{aligned}
& \sum_{t=e_{i}}^{l_{i}} x_{i t}=1, \quad \forall i \\
& \sum_{t=e_{i}}^{l_{i}} t x_{i t}-\sum_{t=e_{j}}^{l_{j}} t x_{j t} \leq d_{i}, \forall j \in P_{i}, i \\
& \sum_{i=1}^{I} r_{i 1} x_{i t} \leq R_{1 t}, \quad \forall t
\end{aligned}
$$




$$
\begin{array}{ll}
\sum_{i=1}^{I} r_{i 2} x_{i t} \leq R_{2 t}, & \forall t \\
\sum_{i=1}^{I} r_{i 3} x_{i t} \leq R_{3 t}, & \forall t \\
\sum_{i=1}^{I} r_{i 4} x_{i t} \leq R_{4 t}, & \forall t \\
x_{i t}=\{0,1\}, \quad & \forall i, t
\end{array}
$$

where $R_{1 t}=R_{2 t}=R_{3 t}=R_{4 t}=10$ 。 The optimization results show as follow:

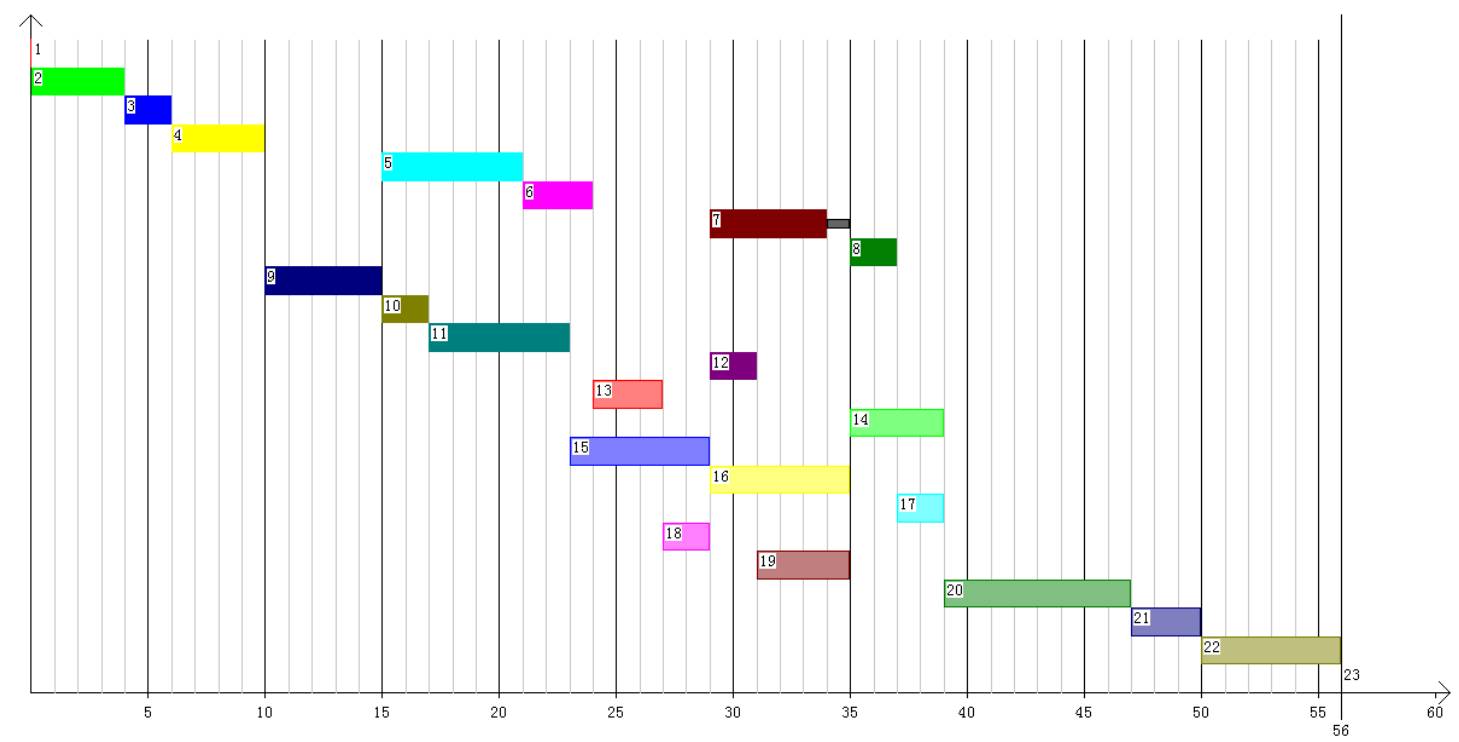

Fig.2 Gantt chart of Optimal results of the project scheduling

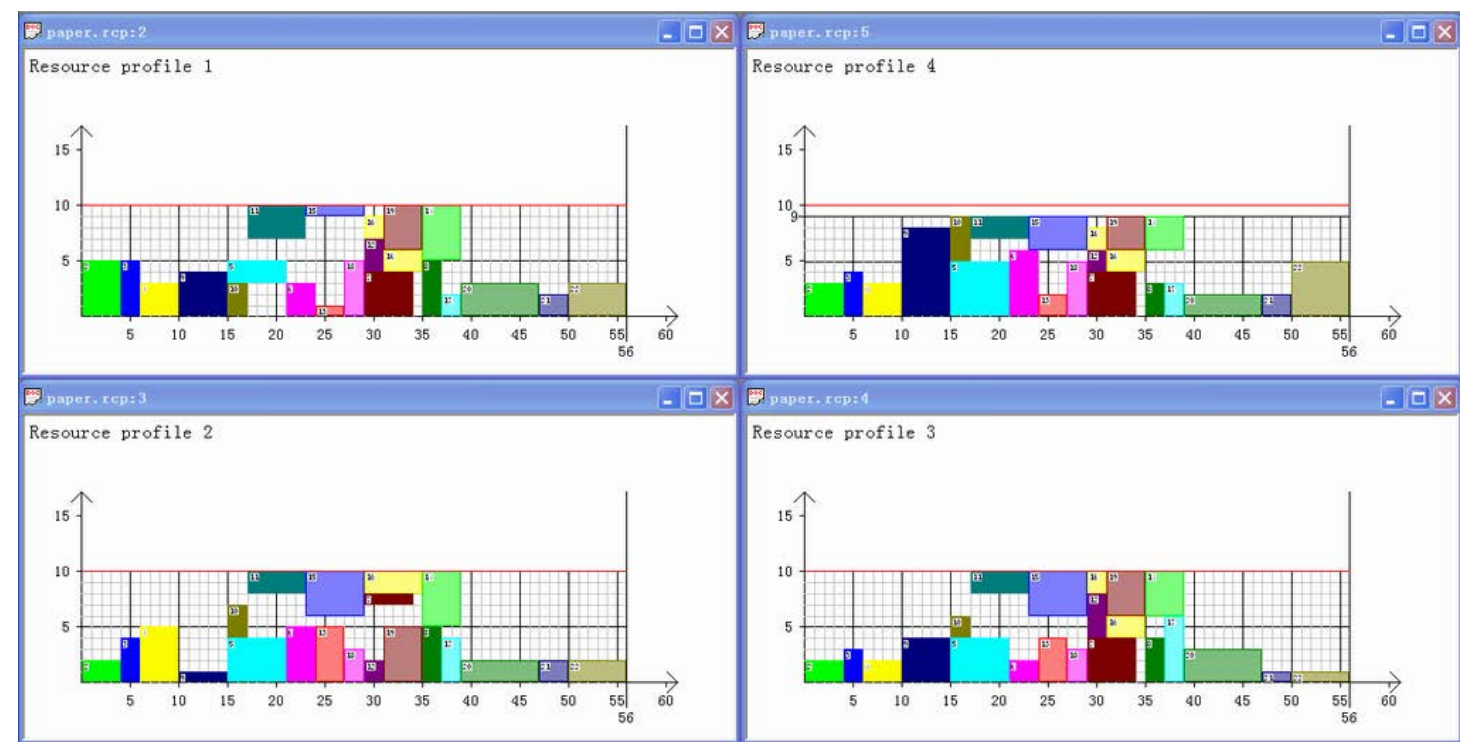

Fig.3 The utilization of project resources

As we can see from figure2 and figure 3, after Tabu Search optimization, the project duration is 56 hours, more than hours before optimization reduces the 29 working hours reduced by $34.12 \%$, the maximum utilization of resources 1 is 10 units, the maximum utilization of resources 2 to 10 units, the maximum use of resources 3 amount of 10 units, the maximum utilization of resources for the nine units 4 , satisfies constraints. Thus, the tabu search algorithm can effectively solve the classic problem RCPSP. 


\section{Conclusion}

This article described the most typical RCPSP problem of resource constrained project scheduling problems, which is also most studied classical, and introduced the mathematical model describes the tabu search algorithm and its algorithm flow, and then took Leopard II tanks' transmission design as example, established the projects scheduling model, adopted tabu search algorithm to solve this problem, demonstrated the effectiveness of tabu search algorithm in solving the classic problem RCPSP.

\section{Acknowledgement}

This work is supposed by National Natural Science Foundation of China (Grant No.61304206).

\section{References}

[1]Information onhttp:// www. peraglobal. com/product_case_list_cont. aspx?one= 3\&two= 19\& three $=99$.

[2] Sprecher A. Resource-Constrained Project Scheduling: Exact Methods for the Multi-mode Case [M].Springer-Verlag, Berlin, 1994.

[3] Viana A. Sousa J. Using met heuristics in multi objective resource constrained Project scheduling [J]. European Journal of Operational Research, Vol.120 (2000), p.359-370.

[4] Patterson J H.A comparison of exact approaches for solving the multiple constrained resource Project scheduling Problem [J].Management Science, Vol.30 (1984), No.7, p.854-867.

[5] R kolisch, A Sprecher. PSPLIB - A project scheduling problem library: OR Software - ORSEP Operations Research Software Exchange Program [J]. European Journal of Operational Research, Vol.1 (1997), No.96, p.205-216.

[6] A Sprecher, R Kolisch, A Drexl. Semi-active, active, and non-delay schedules for the resource-constrained project scheduling problem [J]. European Journal of Operational Research, Vol. 80(1995), p.94-102.

[7] R Klein .PROGRESS: Optimally solving the generalized resource-constrained project scheduling problem. European Journal of Operational Research, Vol.3 (2000) No.127, p.619- 638. 